\title{
Charged radiating stars with Lie symmetries
}

\author{
G. Z. Abebe ${ }^{\mathrm{a}}$, S. D. Maharaj ${ }^{\mathrm{b}}$ \\ Astrophysics and Cosmology Research Unit, School of Mathematics, Statistics and Computer Science, University of KwaZulu-Natal, \\ Private Bag X54001, Durban 4000, South Africa
}

Received: 8 September 2019 / Accepted: 7 October 2019 / Published online: 15 October 2019

(C) The Author(s) 2019

\begin{abstract}
We consider the general model of an accelerating, expanding and shearing radiating star in the presence of charge. Using a new set of variables arising from the Lie symmetries of differential equations we transform the boundary equation into ordinary differential equations. We present several new exact models for a charged gravitating sphere. A particular family of solution may be interpreted as a generalised Euclidean star in the presence of the electromagnetic field. This family admits a linear barotropic equation of state. In the uncharged limit, we regain general relativistic stellar models where proper and areal radii are equal, and its generalisations. Our group theoretical approach selects the physically important cases of Euclidean stars and equations of state.
\end{abstract}

\section{Introduction}

Studying relativistic radiating stars with the kinematical quantities of shear, expansion and acceleration is an important area of research in relativistic astrophysics and cosmology. Such models are important in investigating the physical features of radiating stars such as gravitational collapse, stability, surface luminosity, relaxation causal thermodynamic effects, temperature profiles and particle production at the surface. Several exact models have been investigated in the past where the interior spacetime with the heat flux matches smoothly to the exterior radiating Vaidya spacetime. For some recent investigations considering the physical activities linked with the gravitational collapse and dissipative processes see Sarwe and Tikekar [1], Sharma and Tikekar [2], Reddy et al. [3], Ivanov [4] and Tewari [5,6]. In these and other analyses the interior energy momentum tensor is taken to be a neutral relativistic anisotropic fluid with heat flow. The presence of the electromagnetic field may substantially change the physical features in a relativistic radiating

\footnotetext{
a e-mail: gezahegn@aims.ac.za

be-mail: maharaj@ukzn.ac.za
}

star and will affect the nature of gravitational interactions. For example, Sharif and Azam [7] showed that the electric field reduces the unstable spacetime region during dissipative collapse when studying the dynamical instability of an expansion-free radiating fluid sphere.

The junction conditions matching the interior radiating matter distribution to the Vaidya exterior were completed by Santos [8] for neutral matter. The junction conditions were later generalised to include the electromagnetic field by De Oliveira and Santos [9], Banerjee and Choudhury [10], Tikekar and Patel [11] and Maharaj and Govender [12]. Barreto and Da Silva [13] analysed charged and self-similar distributions and spheres in the diffusion approximations which is valid at the final stages of collapse. Barreto et al. [14] also considered self-similar radiating spheres in the presence of anisotropy in the diffusion limit. A systematic and extensive investigation of nonadiabatic charged spherical gravitational collapse in both the diffusion and free-streaming limits was completed by Di Prisco et al. [15]. Fayos et al. [16] and Fayos and Torres [17] considered the matching of a general spherical spacetime with a charged Vaidya exterior and applied the results to relativistic stars and voids. Barreto and Rosales [18], extending their earlier approach in the diffusion regime, considered charged and self-similar spheres in the free-streaming approximations which is applicable in the early stages of collapse. Cipolletta and Giambo [19] found a class of charged anisotropic collapsing models and demonstrated that the shell focussing singularities may be avoided. The presence of charge in the gravitational collapse delays back hole formation and can even prevent collapse as shown by Pinheiro and Chan [20] by numerically integrating the fluid equations and stellar boundary condition. A similar numerical integration allowed Sharif and Iftikhar [21] to model charged dissipative collapse which highlights the dominate role of the electric field. Thirukkanesh and Govender [22] found a class of exact models by investigating the boundary condition in the presence of charge and shear. These analyses highlight the prominent role that the electro- 
magnetic field plays in the modelling of a radiating star in general relativity.

Charged radiating stars with anisotropic matter interiors have been recently studied by Ivanov [23] for geodesic flows, and also for accelerating matter [24]. In these studies several families of exact solutions have been obtained by transforming the boundary condition to a Riccati using a transformation related to formation of horizons. Here we generate exact solutions for a general charged accelerating, expanding and shearing radiating matter distribution using a symmetry approach. The Lie point symmetry approach has recently been used, under different physical situations, in solving the Einstein field equations for a general relativistic star. The junction condition relating the radial pressure with the heat flux for neutral fluids has been studied with the help of Lie symmetries. Govinder and Govender [25] were the first to study the junction condition in Euclidean stars. Subsequently Abebe et al. [26] analysed a conformally flat radiating star in the absence of charge. Abebe et al. [27] also studied a shearing neutral fluid model in geodesic motion. Recently Abebe et al. [28] considered the junction condition of an accelerating, expanding and shearing stellar in the absence of charge. Note that a comprehensive analysis of shear-free stellar models has been made by Abebe et al. [29] with the help of Lie point symmetries. Other recent treatments with shearing matter utilizing the Lie method include the works of Mohanlal et al. [30], and also [31]. It will be helpful if these investigations could be extended to include the effects of the electromagnetic field.

The main objective of this paper is to generate exact solutions to the boundary condition equation of a charged radiating star using transformations arising from the Lie symmetries of differential equations. In Sect. 2 we briefly discuss the general spherically symmetric spacetime and field equations. We present the junction condition equation for an accelerating, expanding and shearing star with charge. The resulting junction equation is a highly nonlinear partial differential equation which is difficult to solve directly using traditional methods. Thus we use the Lie symmetry approach to transform the partial differential to ordinary differential equations. In Sect. 3 we obtain the Lie point symmetries admitted by the junction condition. We transform the boundary condition to ordinary differential equations using the linear combination of the symmetries. Several explicit solutions are obtained. In Sect. 4 we discuss the role of equation of state and recover the earlier models for uncharged stars. Some concluding remarks are made in Sect. 5.

\section{The model}

The line element determining the interior spacetime of accelerating, expanding and shearing general relativistic radiating stars may be written as

$d s^{2}=-A^{2} d t^{2}+B^{2} d r^{2}+Y^{2}\left(d \theta^{2}+\sin ^{2} \theta d \phi^{2}\right)$,

with metric functions $A=A(r, t), B=B(r, t)$ and $Y=$ $Y(r, t)$. The timelike, unit fluid four-velocity $\mathbf{u}$ is comoving and has the form $u^{a}=\frac{1}{A} \delta_{0}^{a}$. The kinematical quantities have the specific forms

$$
\begin{aligned}
\dot{u}^{a} & =\left(0, \frac{A_{r}}{A B^{2}}, 0,0\right), \\
\Theta & =\frac{1}{A}\left(\frac{B_{t}}{B}+2 \frac{Y_{t}}{Y}\right), \\
\sigma & =-\frac{1}{3 A}\left(\frac{B_{t}}{B}-\frac{Y_{t}}{Y}\right),
\end{aligned}
$$

where $\dot{u}^{a}$ is the acceleration, $\Theta$ is the expansion scalar and $\sigma$ is the magnitude of the shear scalar. Note that subscripts denote differentiation with respect to the coordinates $r$ and $t$.

The energy momentum tensor for the general model has the form

$T_{a b}=(\mu+p) u_{a} u_{b}+p g_{a b}+q_{a} u_{b}+q_{b} u_{a}+\pi_{a b}+E_{a b}$,

where $\mu$ is the density, $p$ is the isotropic pressure, and $q_{a}$ is the heat flux. The anisotropic stress tensor is

$\pi_{a b}=\left(p_{\|}-p_{\perp}\right)\left(n_{a} n_{b}-\frac{1}{3} h_{a b}\right)$,

where $p_{\perp}$ is the radial pressure, $p_{\perp}$ is the tangential pressure, $h_{a b}=u_{a} u_{b}+g_{a b}$ is the projection tensor and $n^{a}=\frac{1}{B} \delta_{1}^{a}$ is a unit radial vector orthogonal to $\mathbf{u}$. Note that the isotropic pressure $p=\frac{1}{3}\left(p_{\|}+2 p_{\perp}\right)$. The tensor $E_{a b}$ is the electromagnetic energy tensor which is given by

$E_{a b}=F_{a}^{c} F_{b c}-\frac{1}{4} F^{c d} F_{c d} g_{a b}$

Since the heat flow is directed radially the vector $\mathbf{q}$ has the form

$q^{a}=(0, q, 0,0)$

and $q^{a} u_{a}=0$.

The Einstein-Maxwell equations are given by

$$
\begin{aligned}
& G_{a b}=T_{a b}, \\
& F_{a b ; c}+F_{b c ; a}+F_{c a ; b}=0, \\
& F_{; c}^{a b}=J^{a},
\end{aligned}
$$

where Faraday's tensor $F_{a b}=\tilde{\phi}_{b ; a}-\tilde{\phi}_{a ; b}$ and the fourcurrent $J^{a}=\epsilon u^{a}$. Note that $\epsilon$ is the proper charge density 
and $\tilde{\phi}_{a}$ is the four-potential. The simplest form for the fourpotential is

$\tilde{\phi}_{a}=(\Phi(r, t), 0,0,0)$,

so that $F_{01}=-F_{10}=-\Phi_{r}$ is the nonzero component of $\mathbf{F}$. From (7) we have

$$
\begin{gathered}
\Phi_{r r}+\left(2 \frac{Y_{r}}{Y}-\frac{B_{r}}{B}-\frac{A_{r}}{A}\right) \Phi_{r}=\epsilon A B^{2}, \\
\left(\frac{1}{A^{2} B^{2}} \Phi_{r}\right)_{t}+\frac{1}{A^{2} B^{2}}\left(\frac{A_{t}}{A}+\frac{B_{t}}{B}\right) \Phi_{r} \\
\quad+\frac{2}{A^{2} B^{2}} \frac{Y_{t}}{Y} \Phi_{r}=0 .
\end{gathered}
$$

On integration we obtain

$$
\begin{aligned}
\Phi_{r} & =\frac{A B}{Y^{2}} l, \\
l(r) & =\int^{r} \epsilon B Y^{2} d r,
\end{aligned}
$$

where $l$ is strictly a function of $r$. Note that we can interpret $l(r)$ as the total charge contained in the sphere up to the radius $r$.

Then the Einstein-Maxwell equations for the line element (1) and the four-potential (8) can be written as the system of nonlinear partial differential equations

$$
\begin{aligned}
\mu+\frac{1}{2} \frac{l^{2}}{Y^{4}}= & \frac{2}{A^{2}} \frac{B_{t}}{B} \frac{Y_{t}}{Y}+\frac{1}{Y^{2}}+\frac{1}{A^{2}} \frac{Y_{t}^{2}}{Y^{2}} \\
& -\frac{1}{B^{2}}\left(2 \frac{Y_{r r}}{Y}+\frac{Y_{r}^{2}}{Y^{2}}-2 \frac{B_{r}}{B} \frac{Y_{r}}{Y}\right), \\
p_{\|}-\frac{1}{2} \frac{l^{2}}{Y^{4}}= & \frac{1}{A^{2}}\left(-2 \frac{Y_{t t}}{Y}-\frac{Y_{t}^{2}}{Y^{2}}+2 \frac{A_{t}}{A} \frac{Y_{t}}{Y}\right) \\
& +\frac{1}{B^{2}}\left(\frac{Y_{r}^{2}}{Y^{2}}+2 \frac{A_{r}}{A} \frac{Y_{r}}{Y}\right)-\frac{1}{Y^{2}}, \\
p_{\perp}+\frac{1}{2} \frac{l^{2}}{Y^{4}}= & -\frac{1}{A^{2}}\left(\frac{B_{t t}}{B}-\frac{A_{t}}{A} \frac{B_{t}}{B}+\frac{B_{t}}{B} \frac{Y_{t}}{Y}\right. \\
& \left.-\frac{A_{t}}{A} \frac{Y_{t}}{Y}+\frac{Y_{t t}}{Y}\right)+\frac{1}{B^{2}}\left(\frac{A_{r r}}{A}\right. \\
& \left.-\frac{A_{r}}{A} \frac{B_{r}}{B}+\frac{A_{r}}{A} \frac{Y_{r}}{Y}-\frac{B_{r}}{B} \frac{Y_{r}}{Y}+\frac{Y_{r r}}{Y}\right), \\
q= & -\frac{2}{A B^{2}}\left(-\frac{Y_{r t}}{Y}+\frac{B_{t}}{B} \frac{Y_{r}}{Y}+\frac{A_{r}}{A} \frac{Y_{t}}{Y}\right), \\
\epsilon= & \frac{l^{\prime}(r)}{B Y^{2}} .
\end{aligned}
$$

The matter variables $\mu, p_{\|}, p_{\perp}, q$, and the proper charge density $\epsilon$ can be determined explicitly once the potential functions $A, B$ and $Y$ are known. The function $l(r)$ determines the charge distribution in the interior spacetime of the star. Equations (11) describe the gravitational and electromagnetic interactions in the interior of an accelerating, expand- ing and shearing charged star with heat flux and anisotropic pressure.

The surface of a spherically symmetric radiating star is the boundary between the interior and the exterior spacetimes. The interior spacetime (1) has to be matched at the surface of the star to the exterior charged Vaidya spacetime

$$
\begin{aligned}
d s^{2}= & -\left(1-\frac{2 m(v)}{R}+\frac{Q^{2}}{R^{2}}\right) d v^{2}-2 d v d R \\
& +R^{2}\left(d \theta^{2}+\sin ^{2} \theta d \phi^{2}\right) .
\end{aligned}
$$

In (12) the function $m(v)$ is the mass of the star and $Q$ is the total charge as measured by an observer at infinity. The metrics (1) and (12) have to be matched at the boundary of the star. The matching of the metrics and the extrinsic curvature at the surface of the star give the junction condition

$\left(p_{\|}\right)_{\Sigma}=(B q)_{\Sigma}$

at the hypersurface $\Sigma$ of the charged radiating sphere. For more information on the matching and junction conditions in the presence of electric field see Maharaj and Govender [12]. From Eqs. (13), (11c) and (11e) we have the junction condition equation

$$
\begin{aligned}
& 4 A B^{2} Y^{3} Y_{t t}+2 A B^{2} Y^{2} Y_{t}^{2}-4 B^{2} Y^{3} A_{t} Y_{t} \\
& -4 A B Y^{3} A_{r} Y_{t}+4 A^{2} B Y^{3} Y_{r t}-4 A^{2} Y^{3} A_{r} Y_{r} \\
& -4 A^{2} Y^{3} B_{t} Y_{r}-2 A^{3} Y^{2} Y_{r}^{2}+2 A^{3} B^{2} Y^{2} \\
& -l^{2} A^{3} B^{2}=0,
\end{aligned}
$$

at the boundary of the star. The charged boundary condition (14) is similar to the uncharged condition derived by Ivanov [24] and Thirukkanesh et al. [32]. When we set $l=0$ then we regain the result of [32]. However the presence of charge makes equation (14) very complicated. It is for this reason that very few exact solutions to (14) have been found when $l \neq 0$. As the equation (14) holds at the boundary $\Sigma$ we treat $l$ as a constant quantity in the integration process; clearly in the interior of the star $l=l(r)$.

\section{Exact solutions}

The method of applying Lie point symmetries in a group theoretical approach has proved to be very successful in generating new exact solutions for radiating stars in relativistic astrophysics. Some results are contained in analyses involving conformally flat stars [26], geodesic stars [27], generalised Euclidean stars [28] and shear-free stars with equation of state [29]. 
It is relatively straight forward to show that (14) admits the Lie symmetries

$$
\begin{aligned}
& X_{1}=A \beta^{\prime}(t) \frac{\partial}{\partial A}-\beta(t) \frac{\partial}{\partial t}, \\
& X_{2}=B \alpha^{\prime}(r) \frac{\partial}{\partial B}-\alpha(r) \frac{\partial}{\partial r},
\end{aligned}
$$

where $\beta(t)$ and $\alpha(r)$ are arbitrary functions. These symmetries may be used to generate group invariant solutions. With the linear combination of these symmetries

$$
\begin{aligned}
a X_{2}-b X_{1}= & -b A \beta^{\prime}(t) \frac{\partial}{\partial A}+a B \alpha^{\prime}(r) \frac{\partial}{\partial B} \\
& +b \beta(t) \frac{\partial}{\partial t}-a \alpha(r) \frac{\partial}{\partial r},
\end{aligned}
$$

we can find the invariants from the conditions

$\frac{d t}{b \beta(t)}=\frac{d r}{-a \alpha(r)}=\frac{d A}{-b A \beta^{\prime}(t)}=\frac{d B}{a B \alpha^{\prime}(r)}=\frac{d Y}{0}$.

These invariants are

$x=\frac{1}{b} \int^{t} \frac{d t}{\beta(t)}+\frac{1}{a} \int^{r} \frac{d r}{\alpha(r)}$,

$A=\frac{f(x)}{\beta(t)}$,

$B=\frac{g(x)}{\alpha(r)}$,

$Y=h(x)$,

for the generator $b X_{1}-a X_{2}$. Here $f, g$ and $h$ are arbitrarily functions of the variable $x$. With the transformation (18), equation (14) becomes

$$
\begin{aligned}
& g^{\prime}+\left(\frac{f^{\prime}}{f}-\frac{h^{\prime \prime}}{h^{\prime}}\right) g \\
& +\left(\left[a \left(4 h^{3} h^{\prime} f^{\prime}-2 h^{2}\left(2 h h^{\prime \prime}+h^{\prime 2}\right) f\right.\right.\right. \\
& \left.\left.\left.\quad+b^{2}\left(l^{2}-2 h^{2}\right) f^{3}\right)\right]\left[4 b f^{2} h^{3} h^{\prime}\right]^{-1}\right) g^{2} \\
& \quad+\left(\frac{b\left(2 h f^{\prime}+f h^{\prime}\right)}{2 a h}\right)=0 .
\end{aligned}
$$

This can be written compactly as

$$
g^{\prime}+\xi(x) g+\varphi(x) g^{2}+\psi(x)=0,
$$

where we have set

$$
\begin{aligned}
\xi(x)= & \frac{f^{\prime}}{f}-\frac{h^{\prime \prime}}{h^{\prime}}, \\
\varphi(x)= & {\left[a \left(4 h^{3} h^{\prime} f^{\prime}-2 h^{2}\left(2 h h^{\prime \prime}+h^{\prime 2}\right) f\right.\right.} \\
& \left.\left.+b^{2}\left(l^{2}-2 h^{2}\right) f^{3}\right)\right]\left[4 b f^{2} h^{3} h^{\prime}\right]^{-1}, \\
\psi(x)= & \frac{b\left(2 h f^{\prime}+f h^{\prime}\right)}{2 a h} .
\end{aligned}
$$

Here the primes denote derivatives with respect to the new independent variable $x$. Equation (20) is a Riccati equation in $g$. It is difficult to complete the integration in general. We make assumptions to integrate and produce new exact solutions.

We observe that in the presence of the electric field $l \neq 0$ there are only two Lie point symmetries (15); in the absence of charge three Lie point symmetries arise as shown by Abebe et al. [28]. This leads to a different nonlinear equation (20) governing the evolution of the charged radiating star. When $l=0$ and $a=1$ then we regain the boundary condition for a neutral radiating star in [28].

\subsection{Linear equation}

To progress we set

$\varphi(x)=0$,

in (20). We note that (22) is a Bernoulli equation in $f$ and can be integrated to give

$f=\frac{\sqrt{2} h h^{\prime}}{\sqrt{-2 b^{2} h^{2}-b^{2} l^{2}+k h}}$,

where $k$ is a constant of integration. On substitution (20) becomes

$$
\begin{aligned}
g^{\prime} & -\left(\frac{h^{\prime}\left(k h-2 b^{2} l^{2}\right)}{2 h\left(2 b^{2} h^{2}+b^{2} l^{2}-k h\right)}\right) g \\
& -\left[b \left(2 h h^{\prime \prime}\left(2 b^{2} h^{2}+b^{2} l^{2}-k h\right)+h^{\prime 2}\left(2 b^{2} h^{2}+3 b^{2} l^{2}\right.\right.\right. \\
& -.2 k h))]\left[\sqrt{2} b\left(-2 b^{2} h^{2}-b^{2} l^{2}+k h\right)^{3 / 2}\right]^{-1}=0
\end{aligned}
$$

which is linear in $g$. Equation (24) can be integrated to give

$$
\begin{aligned}
g=\frac{\sqrt{2 b^{2} h^{2}+b^{2} l^{2}-k h}}{h} & \\
& \times\left(\int ^ { x } \left\{\left[b h \left(h^{\prime 2}\left(2 h\left(k-b^{2} h\right)-3 b^{2} l^{2}\right)\right.\right.\right.\right. \\
& \left.\left.-2 h h^{\prime \prime}\left(2 b^{2} h^{2}-k h+b^{2} l^{2}\right)\right)\right]\left[a \left(2 b^{2} h^{2}-k h\right.\right. \\
& \left.\left.\left.+b^{2} l^{2}\right)^{3 / 2} \sqrt{2 h\left(k-2 b^{2} h\right)-2 b^{2} l^{2}}\right]^{-1}\right\} d x \\
& +m)
\end{aligned}
$$

where $m$ is a constant of integration. 
Hence we have the gravitational potentials

$$
\begin{aligned}
A= & \frac{\sqrt{2} h h^{\prime}}{\beta(t) \sqrt{-2 b^{2} h^{2}-b^{2} l^{2}+k h}}, \\
B= & \frac{\sqrt{2 b^{2} h^{2}+b^{2} l^{2}-k h}}{h \alpha(r)} \\
& \times\left(\int ^ { x } \left\{\left[b h \left(h^{\prime 2}\left(2 h\left(k-b^{2} h\right)-3 b^{2} l^{2}\right)\right.\right.\right.\right. \\
& \left.\left.-2 h h^{\prime \prime}\left(2 b^{2} h^{2}-k h+b^{2} l^{2}\right)\right)\right] \\
& \times\left[a\left(2 b^{2} h^{2}-k h+b^{2} l^{2}\right)^{3 / 2}\right. \\
& \left.\left.\left.\sqrt{2 h\left(k-2 b^{2} h\right)-2 b^{2} l^{2}}\right]^{-1}\right\} d x+m\right), \\
Y= & h,
\end{aligned}
$$

$$
B=\frac{\sqrt{h} h^{\prime}}{\alpha(r)\left[m-\int^{x} \frac{a}{4 b k}\left(\frac{b^{2} k^{2}\left(2 h^{2}-l^{2}\right)}{h^{3}}+4 h h^{\prime \prime}+4 h^{\prime 2}\right) d x\right]},
$$

$Y=h$,

which another solution to the master equation.

\subsection{Riccati equation}

Here we set

$\xi(x)=0$,

in (20) for simplicity. Equation (32) is a separable equation and we find that

$f(x)=k h^{\prime}$,

on integration where $k$ is an arbitrary constant. Then on substitution (20) becomes

$g^{\prime}+\frac{a\left(b^{2} k^{2} l^{2}-2\left(b^{2} k^{2}+1\right) h^{2}\right)}{4 b k h^{3}} g^{2}+\frac{b k\left(2 h h^{\prime \prime}+h^{\prime 2}\right)}{2 a h}=0$,

$\psi(x)=0$,

in (20). We observe that (27) is a linear in $f$ and yields

$f(x)=\frac{k}{\sqrt{h}}$,

on integration. Here $k$ is a constant of integration. Substituting (28) into (20) we have

$$
\begin{aligned}
& g^{\prime}+\left(-\frac{h^{\prime}}{2 h}-\frac{h^{\prime \prime}}{h^{\prime}}\right) g \\
& -\frac{a\left(2 b^{2} k^{2} h^{2}-b^{2} k^{2} l^{2}+4 h^{4} h^{\prime \prime}+4 h^{3} h^{\prime 2}\right)}{4 b k h^{7 / 2} h^{\prime}} g^{2}=0,
\end{aligned}
$$

which is a Bernoulli equation in $g$. It can be integrated to yield

$$
g=\frac{\sqrt{h} h^{\prime}}{m-\int^{x} \frac{a}{4 b k}\left(\frac{b^{2} k^{2}\left(2 h^{2}-l^{2}\right)}{h^{3}}+4 h h^{\prime \prime}+4 h^{\prime 2}\right) d x},
$$

where $m$ is a constant of integration.

Therefore we have the metric functions

$$
A=\frac{k}{\beta(t) \sqrt{h}},
$$

which is a simpler Riccati equation in $g$. Equation (34) is difficult to integrate in general. Abebe et al. [28] demonstrated a solution for a neutral fluid $(l=0)$ for a particular form of the function $h$.

\subsection{Case: $f(x)=\operatorname{cg}(x)$}

Another Bernoulli equation can obtained if we set

$f=c g$,

where $c$ is an arbitrary constant. Then (20) becomes

$$
\begin{aligned}
& g^{\prime}+\left(\frac{(b c-a) h^{\prime 2}-2 a h h^{\prime \prime}}{2 h(b c+a) h^{\prime}}\right) g \\
& \quad+\left(\frac{b^{2} a^{2} c^{2}\left(l^{2}-2 h^{2}\right)}{4 h^{3}(b c+a)^{2} h^{\prime}}\right) g^{3}=0,
\end{aligned}
$$

which is a Bernoulli equation in $g$. Although $h$ is an unknown function of the new variable $x$ we can integrate (36) to obtain

$$
\begin{aligned}
& g(x)= \\
& \times \frac{h^{\frac{a-b c}{2 b c+2 a}} h^{\prime \frac{a}{b c+a}}}{\left(\frac{b^{2} a^{2} c^{2}}{2(b c+a)^{2}} \int^{x}\left(l^{2}-2 h^{2}\right) \frac{h^{\prime} \frac{a-b c}{b c+a}}{h^{\frac{2 a+4 b c}{b c+a}}} d x+d\right)^{1 / 2}},
\end{aligned}
$$

where $d$ is a constant of integration. Thus we have found an exact solution to the boundary condition (14). 
The solution is expressible in terms of the function $h$ which is arbitrary. Thus the potential functions become

$$
\begin{aligned}
& A=\frac{c^{\frac{a-b c}{2 b c+2 a}} h^{\prime \frac{a}{b c+a}}}{\beta(t)\left(\frac{b^{2} a^{2} c^{2}}{2(b c+a)^{2}} \int^{x}\left(l^{2}-2 h^{2}\right) \frac{h^{\prime} \frac{a-b c}{b c+a}}{h^{\frac{2 a+4 b c}{b c+a}}} d x+d\right)^{1 / 2}} \\
& B=\frac{h^{\frac{a-b c}{2 b c+2 a}} h^{\prime} \frac{a}{b c+a}}{\alpha(r)\left(\frac{b^{2} a^{2} c^{2}}{2(b c+a)^{2}} \int^{x}\left(l^{2}-2 h^{2}\right) \frac{h^{\prime} \frac{a-b c}{b c+a}}{h^{\frac{2 a+4 b c}{b c+a}}} d x+d\right)^{1 / 2}}
\end{aligned}
$$

$Y=h$,

which is a solution to the master equation (14). Note that $h$ is an arbitrary function of the variable $x=\frac{1}{b} \int^{t} \frac{d t}{\beta(t)}+$ $\frac{1}{a} \int^{r} \frac{d r}{\alpha(r)}$. This solution consists of the arbitrary functions $\alpha(r), \beta(t)$, and arbitrary constants $a, b, c$ and $d$. This may help us to generate infinitely many solutions for particular choices of these functions and constants.

\section{Equation of state}

The solutions found in this paper are physically reasonable. An important physical feature for an astrophysical object is an equation of state. The potentials in (38) produce this feature. For this case the Einstein-Maxwell field equations for the interior matter distribution become

$$
\begin{aligned}
& \mu=\frac{b^{2} c^{2}+b a c+a^{2}}{b a c}\left[\frac{2(a-b c)\left(h h^{\prime \prime}+h^{\prime 2}\right) \zeta(x)}{b a c(b c+a) h^{\frac{3 a+b c}{b c+a}} h^{\prime} \frac{2 a}{b c+a}}\right. \\
& \left.+\frac{\operatorname{bac}\left(2 h^{2}-l^{2}\right)}{(b c+a)^{2} h^{4}}\right] \text {, } \\
& p_{\|}=\frac{2(b c-a)\left(h h^{\prime \prime}+h^{\prime 2}\right) \zeta(x)}{b a c(b c+a) h^{\frac{3 a+b c}{b c+a}} h^{\prime} \frac{2 a}{b c+a}}+\frac{b a c\left(l^{2}-2 h^{2}\right)}{(b c+a)^{2} h^{4}}, \\
& p_{\perp}=\frac{b^{2} a^{2} c^{2}(b c-a)\left(l^{2}-2 h^{2}\right)^{2}}{8(a+b c)^{3} h^{\frac{5 a+7 b c}{a+b c}} h^{\prime} \frac{2 b c}{a+b c} \zeta(x)}+\frac{(a-b c)^{2} l^{2} h^{\prime \prime}}{4(a+b c)^{2} h^{3} h^{\prime 2}} \\
& +\left[( b c - a ) \zeta ( x ) \left((b c-a) h^{\prime 4}+(3 a+b c) h h^{\prime \prime} h^{\prime 2}\right.\right. \\
& \left.\left.+2 a h^{2} h^{(3)} h^{\prime}-2 a h^{2} h^{\prime \prime 2}\right)\right]\left[2 b^{2} a^{2} c^{2} h^{\frac{3 a+b c}{a+b c}}\right. \\
& \left.\times h^{\prime \frac{4 a+2 b c}{a+b c}}\right]^{-1}+\frac{b c(a-b c)}{(a+b c)^{2} h^{2}}-\frac{l^{2}}{2 h^{4}} \\
& -\frac{(a-b c)(a+2 b c) l^{2}}{2(a+b c)^{2} h^{4}}-\frac{(a-b c)^{2} h^{\prime \prime}}{2(a+b c)^{2} h h^{\prime 2}}, \\
& q=\frac{\zeta(x) \alpha(r)}{b a c(b c+a)^{2} h^{\frac{3 a}{b c+a}+\frac{7}{2}} h^{\prime} \frac{3 a}{b c+a}}\left(b^{2} a^{2} c^{2}\left(l^{2}-2 h^{2}\right)\right. \\
& \times h^{\frac{2 a}{b c+a}} h^{\prime} \frac{2 a}{b c+a}-2 \zeta(x) h^{3}(a-b c)(b c+a)
\end{aligned}
$$

$$
\begin{gathered}
\left.\times\left(h h^{\prime \prime}+h^{\prime 2}\right)\right), \\
\epsilon=\frac{\alpha(r) l^{\prime}(r)}{h^{\frac{3 b c+5 a}{2(b c+a)}} h^{\prime} \frac{a}{b c+a}} \zeta(x)^{1 / 2},
\end{gathered}
$$

where we set $\zeta(x)=d+\frac{b^{2} a^{2} c^{2}}{2(b c+a)^{2}} \int^{x}\left(l^{2}-2 h^{2}\right) \frac{h^{\prime} \frac{a-b c}{b c+a}}{h^{\frac{2 a+4 b c}{b c+a}}} d x$. We observe that

$p_{\|}=\lambda \mu, \quad \lambda=-\frac{b a c}{b^{2} c^{2}+b a c+a^{2}}$.

Therefore the charged stellar model (39) satisfies a linear equation of state (40) which barotropic. It is remarkable that an equation of state exists even though $h(x)$ is an arbitrary function; we are free to select any analytic form for $h$. The Lie theory of differential equations has generated a rich family of of new solutions for radiating charged stellar models which satisfies a barotropic equation of state.

If we omit the charge, $(l=0)$ and set $a=1$ in (38) then we have

$$
\begin{aligned}
& A=\frac{c h^{\frac{1-b c}{2(b c+1)}} h^{\prime \frac{1}{b c+1}}}{\beta(t)\left(d-\frac{b^{2} c^{2}}{(b c+1)^{2}} \int_{1}^{x} h^{\frac{-2 b c}{b c+1}} h^{\prime \frac{1-b c}{b c+1}} d x\right)^{1 / 2}}, \\
& B=\frac{h^{\frac{1-b c}{2(b c+1)}} h^{\prime} \frac{1}{b c+1}}{\alpha(r)\left(d-\frac{b^{2} c^{2}}{(b c+1)^{2}} \int_{1}^{x} h^{\frac{-2 b c}{b c+1}} h^{\prime \frac{1-b c}{b c+1}} d x\right)^{1 / 2}}, \\
& Y=h,
\end{aligned}
$$

The model (41) for an uncharged generalised Euclidean star was obtained by Abebe et al. [28]. In an Euclidean star the gravitational potentials are related by $B=Y_{r}$ which was first introduced by Herrera and Santos [33]. Therefore our model (38) may be interpreted as a charged generalised Euclidean star. Note that the particular Govinder and Govender [25] solution

$$
\begin{aligned}
& A=\frac{x^{n-1}}{\beta(t)}, \\
& B=k n \frac{x^{n-1}}{\alpha(r)}, \\
& Y=k x^{n}, \quad \text { where } x=\int \frac{d t}{\beta(t)}+\int \frac{d r}{\alpha(r)},
\end{aligned}
$$

where $k^{2} n^{3}-2 k n^{2}+2 n(k-1)+2=0$ is contained in (41) if we set the functional value $h(x)=k x^{n}$. The solution (42) is an example of an Euclidean general relativistic star. Note that the particular uncharged models (41) and (42) also admit an equation of state; these can be regained from our general equation of state (40). The various possible models with equation of state are given in Table 1 . It is interesting to note that separable metric potentials also yield an equation of state as shown by Abebe and Maharaj [34] 
Table 1 Stars, metric functions and equations of state

\begin{tabular}{|c|c|c|}
\hline Star & Metric functions & Equation of state \\
\hline \multirow[t]{3}{*}{ Charged generalised Euclidean star } & $A=\frac{\frac{a-b c}{2 b c+2 a} h^{\prime} \frac{a}{b c+a}}{\beta(t)\left(\frac{b^{2} a^{2} c^{2}}{2(b c+a)^{2}} \int^{x}\left(l^{2}-2 h^{2}\right) \frac{h^{\prime} \frac{a-b c}{b c+a}}{h^{\frac{2 a+4 b c}{b c+a}}} d x+d\right)^{1 / 2}}$ & $p_{\|}=\lambda \mu$ \\
\hline & $B=\frac{h^{\frac{a-b c}{2 b c+2 a}} h^{\prime} \frac{a}{b c+a}}{\alpha(r)\left(\frac{b^{2} a^{2} c^{2}}{2(b c+a)^{2}} \int^{x}\left(l^{2}-2 h^{2}\right) \frac{h^{\prime} \frac{a-b c}{b c+a}}{h^{\frac{2 a+4 b c}{b c+a}}} d x+d\right)^{1 / 2}}$ & $\lambda=-\frac{b a c}{b^{2} c^{2}+b a c+a^{2}}$ \\
\hline & $Y=h(x)$ & \\
\hline \multirow[t]{3}{*}{ Uncharged generalised Euclidean star } & $A=\frac{c h^{\frac{1-b c}{2(b c+1)}} h^{\prime} \frac{1}{b c+1}}{\beta(t)\left(d-\frac{b^{2} c^{2}}{(b c+1)^{2}} \int_{1}^{x} h^{-2 b c} \frac{-2 b c}{b c+1} h^{\prime} \frac{1-b c}{b c+1} d x\right)^{1 / 2}}$ & $p_{\|}=\lambda \mu$ \\
\hline & $B=\frac{\frac{1-b c}{2(b c+1)} h^{\prime} \frac{1}{b c+1}}{\alpha(r)\left(d-\frac{b^{2} c^{2}}{(b c+1)^{2}} \int_{1}^{x} h^{\frac{-2 b c}{b c+1}} h^{\prime \frac{1-b c}{b c+1}} d x\right)^{1 / 2}}$ & $\lambda=-\frac{b c}{b^{2} c^{2}+b c+1}$ \\
\hline & $Y=h(x)$ & \\
\hline \multirow[t]{3}{*}{ Euclidean star } & $A=\frac{x^{n-1}}{\beta(t)}$ & $p_{\|}=\lambda \mu$ \\
\hline & $B=k n \frac{x^{n-1}}{\alpha(r)}$ & $\lambda=\frac{2 n-k^{2} n^{3}-2}{2 k^{2} n^{3}-2 k^{2} n^{2}}$ \\
\hline & $Y=k x^{n}$ provided that $k^{2} n^{3}-2 k n^{2}+2 n(k-1)+2=0$ & \\
\hline
\end{tabular}

\section{Conclusion}

Earlier studies involving group theoretical approaches to study bounded matter distributions were completed in the absence of charge. In this study we have attempted to include the effect of the electric field in the stellar model. We studied the junction condition of an accelerating, expanding and shearing radiating star in the presence of charge. We found that the boundary equation admits two Lie point symmetries. In the uncharged limit there are three Lie point symmetries; only two symmetries have the consequence of producing a completely different form for the boundary equation, in the presence of charge, which is difficult to analyse. Using the two Lie symmetries we transformed the partial differential equations into ordinary differential equations with new variables. We solved the transformed ordinary equation and produced new solutions in terms of the original variables.We showed that one family of solutions admits a linear barotropic equation of state. We can interpret this family as a charged generalised Euclidean star model. The uncharged Euclidean and generalised Euclidean models of Govinder and Govender [25] and Abebe et al. [28] respectively were regained in the uncharged limit. Our analysis in the presence of the electromagnetic field shows the relevance of using a group theoretic approach in solving problems with the electromagnetic field. It is interesting to observe that the group theoretical approach selects important physical models, namely Euclidean stars and the equation of state.

Acknowledgements GZA thanks the University of KwaZulu-Natal for financial support. SDM acknowledges that this research is supported by the South African Research Chair Initiative of the Department of Science and Technology and the National Research Foundation.

Data Availability Statement This manuscript has no associated data or the data will not be deposited. [Authors' comment: There is no associated data for this manuscript.]

Open Access This article is distributed under the terms of the Creative Commons Attribution 4.0 International License (http://creativecomm ons.org/licenses/by/4.0/), which permits unrestricted use, distribution, and reproduction in any medium, provided you give appropriate credit to the original author(s) and the source, provide a link to the Creative Commons license, and indicate if changes were made. Funded by SCOAP $^{3}$.

\section{References}

1. S. Sarwe, R. Tikekar, Int. J. Mod. Phys. D 19, 1889 (2010)

2. R. Sharma, R. Tikekar, Gen. Relativ. Gravit. 44, 2503 (2012)

3. K.P. Reddy, M. Govender, S.D. Maharaj, Gen. Relativ. Gravit. 47, 35 (2015)

4. B.V. Ivanov, Int. J. Mod. Phys. D 25, 1650049 (2016)

5. B.C. Tewari, Astrophys. Space Sci. 342, 73 (2012)

6. B.C. Tewari, Gen. Relativ. Gravit. 45, 1547 (2013)

7. M. Sharif, M. Azam, Gen. Relativ. Gravit. 44, 1181 (2012)

8. N.O. Santos, Mon. Not. R. Astron. Soc. 216, 403 (1985)

9. A.K.G. de Oliveira, N.O. Santos, Mon. Not. R. Astron. Soc. 312, 640 (1987)

10. A. Banerjee, S.B. Choudhury, Gen. Relativ. Gravit. 21, 785 (1989)

11. R. Tikekar, L.K. Patel, K. Pramana, J. Phys. 39, 17 (1992)

12. S.D. Maharaj, M. Govender, K. Pramana, J. Phys. 54, 715 (2000)

13. W. Barreto, A. Da Silva, Class. Quantum Grav. 16, 1783 (1999)

14. W. Barreto, B. Rodriguez, L. Rosales, O. Serrano, Gen. Relativ. Gravit. 39, 23 (2007)

15. A. Di Prisco, L. Herrera, G. Le Denmat, M.A.H. MacCallum, N.O. Santos, Phys. Rev. D 76, 064017 (2007) 
16. F. Fayos, J.M.M. Senovilla, R. Torres, Class. Quantum Grav. 20, 2579 (2003)

17. F. Fayos, R. Torres, Class. Quantum Grav. 21, 1351 (2004)

18. W. Barreto, L. Rosales, Gen. Relativ. Gravit. 43, 2833 (2011)

19. F. Cipolletta, R. Giambo, Class. Quantum Grav. 29, 245008 (2012)

20. G. Pinheiro, R. Chan, Gen. Relativ. Gravit. 45, 243 (2013)

21. M. Sharif, S. Iftikhar, Astrophys. Space Sci. 357, 79 (2015)

22. S. Thirukkanesh, M. Govender, Int. J. Mod. Phys. D 22, 1350087 (2013)

23. B.V. Ivanov, Eur. Phys. J. C 79, 255 (2019)

24. B.V. Ivanov, Eur. Phys. J. C 79, 520 (2019)

25. K.S. Govinder, M. Govender, Gen. Relativ. Gravit. 44, 147 (2012)

26. G.Z. Abebe, K.S. Govinder, S.D. Maharaj, Int. J. Theor. Phys. 52, 3244 (2013)

27. G.Z. Abebe, S.D. Maharaj, K.S. Govinder, Gen. Relativ. Gravit. 46, 1650 (2014)
28. G.Z. Abebe, S.D. Maharaj, K.S. Govinder, Gen. Relativ. Gravit. 46, 1733 (2014)

29. G.Z. Abebe, S.D. Maharaj, K.S. Govinder, Eur. Phys. J. C 75, 496 (2015)

30. R. Mohanlal, S.D. Maharaj, A.K. Tiwari, R. Narain, Gen. Relativ. Gravit. 48, 87 (2016)

31. R. Mohanlal, R. Narain, S.D. Maharaj, J. Math. Phys. 58, 072503 (2017)

32. S. Thirukkanesh, S. Rajah, S.D. Maharaj, J. Math. Phys. 53, 632506 (2012)

33. L. Herrera, N.O. Santos, Gen. Relativ. Gravit. 42, 2383 (2010)

34. G.Z. Abebe, S.D. Maharaj, K. Pramana, J. Phys. 88, 19 (2017) 